\title{
Magnetofluidic spreading in circular chambers under a uniform magnetic field
}

Mohammad Amin Maleki, ,"\# Jun Zhang ${ }^{2, \#}$, Navid Kashaninejad ${ }^{2, \#}$, M. Soltani, 1,3,4,*, Nam-Trung Nguyen, ${ }^{2, *}$

${ }^{1}$ Department of Mechanical Engineering, K. N. Toosi University of Technology, Tehran 19697, Iran

${ }^{2}$ Queensland Micro- and Nanotechnology Centre and ${ }^{\ddagger}$ School of Natural Science, Griffith University, Nathan Campus, 170 Kessels Road, Brisbane, QLD 4111, Australia

${ }^{3}$ Department of Electrical and Computer Engineering, University of Waterloo, ON N2L 3G1, Canada

${ }^{4}$ Centre for Biotechnology and Bioengineering (CBB), University of Waterloo, Waterloo, ON N2L 3G1, Canada

\# These authors contributed equally to this work.

*Correspondence: madsoltani@uwaterloo.ca; nam-trung.nguyen@griffith.edu.au

\begin{abstract}
Elucidating the microscale interaction between magnetism and fluid flow is of great importance for designing micro-magnetofluidic gradient generators, micromixers, and particle sorters. Coflowing magnetic and non-magnetic fluids can lead to instability at their interface and subsequent rapid mixing. The mismatch in the magnetisation of the fluids leads to instabilities. The present study systematically investigates the magnetofluidic spreading phenomena of both magnetic nanoparticles and non-magnetic fluorescent dye in consecutive circular chambers. Numerical simulations and experimental investigations were conducted to thoroughly evaluate the physics of magnetofluidic spreading. We show that the presence of the consecutive chambers can enhance magnetofluidic spreading by slowing down the flow and increasing the mass transfer rate transversal to the flow direction. The numerical results reveal that the magnetic force, induced by the magnetic susceptibility gradient, generates cross-sectional secondary flows that steer particles toward both the top and bottom walls. The induced secondary flow also enhances the transport of fluorescent dye, thereby leading to a higher mass transfer rate as compared to pure molecular
\end{abstract}


diffusion. The findings provide further insights into the microscale spreading phenomenon of magnetic and non-magnetic particles in a magnetic field.

Keywords: Microfluidics, Magnetofluidic spreading, Numerical modelling, circular chambers, ferrofluid, Micro-magnetofluidic gradient generators

\section{Introduction}

Continuous-flow microfluidics enables manipulation of particles in a fluid. Passive manipulation methods depend merely on hydrodynamic forces (Moghadas et al. 2018), while active methods employ external sources such as acoustic (Marzo et al. 2015), electrical (Tajik et al. 2019), optical (Diekmann et al. 2016), thermal (D'Eramo et al. 2018) and magnetic (Munaz et al. 2018) actuators. Among active methods, magnetic manipulation has attracted considerable attention because of its high efficiency, cost-effectiveness and straightforward implementation (Gao et al. 2019). Micromagnetofluidics refers to the science and technology that combines magnetism with microfluidics to gain new functionalities and capabilities (Nguyen 2012). Micro-magnetofluidic platforms provide contactless manipulation of both magnetic and nonmagnetic particles and have promising applications such as cell cytometry (Knowlton et al. 2017), point-of-care diagnostics (van Reenen et al. 2014), cell analysis (Chen et al. 2014), and immunological assays (Gaster et al. 2009).

Depending on the size of magnetic particles, magnetic fluids can be categorized as magnetorheological fluid and ferrofluid (Nguyen 2012). If magnetic particles dispersed in the carrier fluid are larger than $10 \mathrm{~nm}$, the fluid is called magnetorheological fluid as the viscosity of the fluid can be tuned by the magnetic field. In a ferrofluid, which is the main scope of the current manuscript, the fluid is a stable suspension of magnetic nanoparticles with diameters on the order of $10 \mathrm{~nm}$ or smaller. The stability of the magnetic colloid relies on the thermal energy and the balance between attractive (dipole-dipole and Van der Waals) and repulsive (electrostatic and steric) interactions. The stability of ferrofluid colloidal with magnetic particles of $\sim 10 \mathrm{~nm}$ is mainly supported by Brownian motion, electrostatic repulsion, and steric repulsion (Rosensweig 1979). Thus, the magnetic nanoparticles disperse well in the carrier fluid, and ferrofluid as the whole behaves like a paramagnetic liquid (Zhao et al. 2016).

Ferrofluid has been employed in micro magnetofluidic systems for applications such as labelfree separation of particles(Kose and Koser 2012; Zhang et al. 2016; Zhao et al. 2016; Zhu et al. 2014; Zhu et al. 2012) and micro-mixing (Hejazian et al. 2016; Zhu and Nguyen 2012b). 
Unravelling the microscale interaction between magnetism and fluid flow is of great importance for designing microfluidic devices. Co-flowing of magnetic and non-magnetic fluids is the most popular configuration that can lead to instability at the interface and subsequent rapid mixing. Several studies have been conducted to investigate magnetofluidic spreading of ferrofluid under a uniform magnetic field. Wang et al. (Wang et al. 2015a; Wang et al. 2015b) numerically and experimentally studied the spreading phenomenon of ferrofluid in a straight channel, following the earlier work in this field (Zhu and Nguyen 2012a). The authors systematically investigated the effects of magnetic field, concentration of the magnetic particles, flow rate, viscosity and diameter of the particle as well as flow rate ratio on magnetic spreading. The results indicated that magnetofluidic spreading is mainly caused by secondary convective flow. Our earlier work reported the magnetofluidic spreading phenomenon for the two cases of ferrofluid core and ferrofluid cladding (Zhu and Nguyen 2012a). We found that the spreading effect increases with magnetic field strength. Spreading of ferrofluid-core configuration is more sensitive to the magnetic field than the ferrofluid-cladding configuration. Subsequently, we investigated ferrofluid mixing under a uniform magnetic field in a circular microchamber (Zhu and Nguyen 2012b). In this study, ferrofluid served as the core stream surrounded by two diamagnetic cladding streams. The proposed device led to a mixing efficiency of up to $90 \%$.

However, the effects of consecutive circular chambers on ferrofluid spreading as well as the influence of ferrofluid on the transport of nonmagnetic fluid in circular chambers remain unknown. In the present work, we systematically investigate magnetofluidic spreading of ferrofluid and dissolved fluorescent dye over a series of circular microchambers. Numerical modelling and experimental investigations are carried out to characterise the magnetofluidic spreading phenomenon. The numerical data agree well with the experimental results. In addition, we explore the effects of the flow rate ratio and the magnetic field strength on the spreading phenomenon. This study provides further insights regarding the spreading behaviours of magnetic and nonmagnetic nanoparticles in the microscale under a uniform magnetic field.

\section{Materials and experimental method}

\subsection{Design and fabrication of the micro-magnetofluidic spreading system}

Figure 1a shows the micro-magnetofluidic spreading system investigated in the present study. The system consists of an electromagnet that induces a uniform magnetic field across the microfluidic 
device positioned in the air gap. The flux density in the air gap of the electromagnet can be adjusted by the applied current, Figure 1b. The microfluidic device was designed as a straight channel with six circular chambers, Figure 1c, d. The gap $S$ between two adjacent chambers is $520 \mu \mathrm{m}$. The width $W$ of the straight channel is $200 \mu \mathrm{m}$. The circular chambers have a diameter of $1 \mathrm{~mm}$. The microchannels and chambers have a height $h$ of $50 \mu \mathrm{m}$. The total length $L$ of the microfluidic device is $9.37 \mathrm{~mm}$. Figure 1c schematically illustrates the above geometrical parameters of the microfluidic device. The microfluidic device was fabricated using the standard photolithography and soft lithography with polydimethylsiloxane (PDMS) (Kashaninejad et al. 2012). The PDMS device with the microchannels was bonded to another PDMS slab after 1.5 minutes of surface treatment with oxygen plasma. The width of the PDMS microfluidic device was carefully cropped to fit the air gap dimension of the custom-made electromagnet.

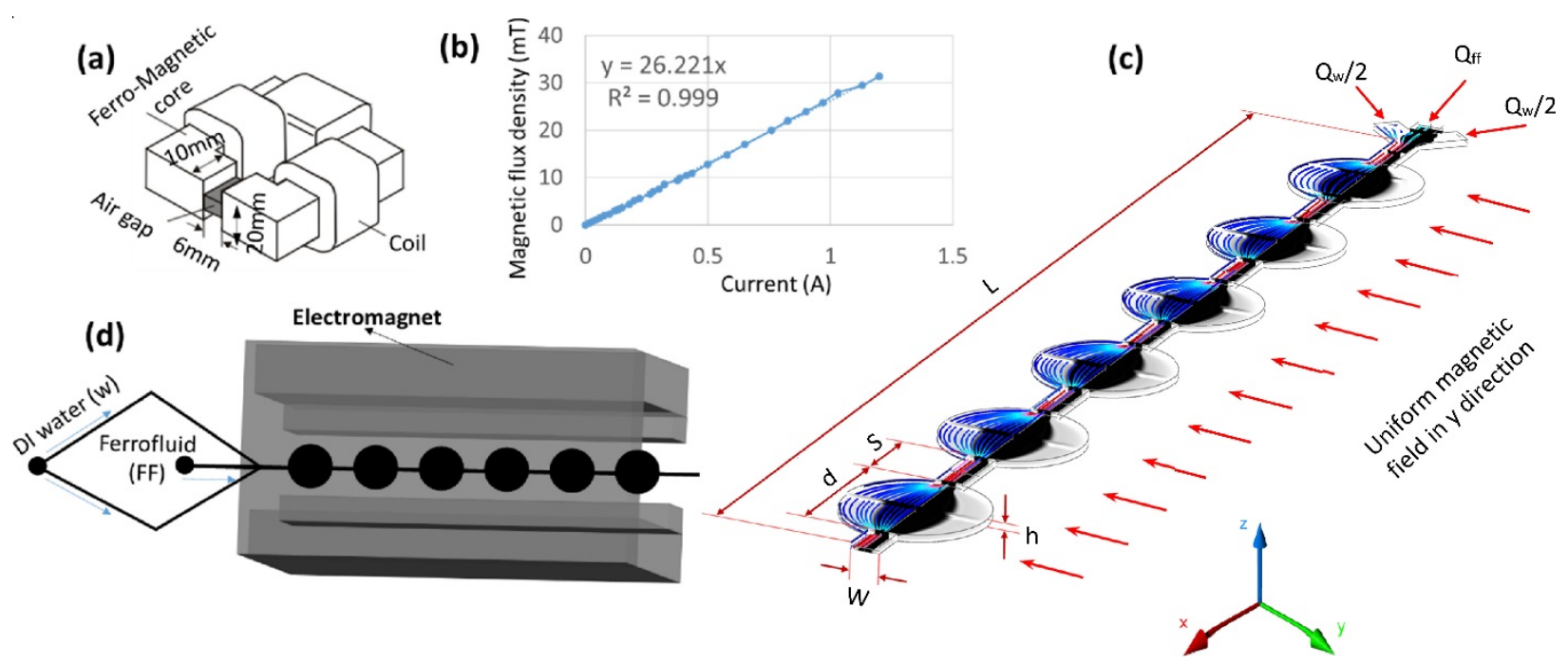

Fig. 1 Magnetofluidic spreading in circular chambers. (a) The U-shaped electromagnet; (b) The magnetic flux density of the electromagnet versus the applied electrical current; (c) The schematic of magnetofluidic spreading of magnetic nanoparticles under a uniform magnetic field with coflow configuration of ferrofluid and DI water $(\mathrm{L}=9.37 \mathrm{~mm} ; \mathrm{W}=200 \mu \mathrm{m} ; \mathrm{h}=50 \mu \mathrm{m} ; \mathrm{S}=520 \mu \mathrm{m}$; $\mathrm{d}=1 \mathrm{~mm}$ ). $\mathrm{Q}_{\mathrm{ff}}$ and $\mathrm{Q}_{\mathrm{w}}$ refer to the flow rate of ferrofluid and DI water, respectively. (d) The microfluidic device inserted at the air gap of the electromagnet.

\subsection{Preparation of ferrofluid and fluorescent dye}

In the present work, we used a diluted commercial water-based ferrofluid that was purchased from Ferrotec company (Nashua, NH, USA; Catalog No. EMG-707). According to the manufacturer's information, the nominal size of the nanoparticles is $10 \mathrm{~nm}$. The EMG-707 ferrofluid consists of 
magnetite iron oxide nanoparticles, suspended in DI water with an anionic surfactant to prevent aggregation. The volume ratio of iron oxide nanoparticles in the ferrofluid is $2 \%$. The saturation magnetisation and the density of the ferrofluid are $11 \mathrm{mT}$ and $1,100 \mathrm{~kg} / \mathrm{m}^{3}$, respectively.

To prepare the fluorescent dye, which was representative of non-magnetic fluid, $0.5 \% \mathrm{w} / \mathrm{v}$ of fluorescein sodium salt (Sigma-Aldrich, Product No. F6377) was dissolved in Milli-Q ${ }^{\circledR}$ ultrapure water (Milli-Q ${ }^{\circledR}$ Direct 8 water purification system with a specific resistance of $18.2 \mu \Omega$ and $0.22 \mu \mathrm{m}$ membrane filter). Subsequently, $1 \mathrm{ml}$ of EMG-707 ferrofluid solution was further diluted with $2 \mathrm{ml}$ of Milli- $\mathrm{Q}^{\circledR}$ water to achieve the desired concentration of magnetic particles.

\subsection{Experimental setup and data analysis}

A custom-made U-shaped electromagnet was fabricated to induce a uniform magnetic field across the microfluidic device, Figure 1a. Before the experiments, the magnetic flux density of the electromagnet was calibrated with the applied electric current, Figure 1b. The microfluidic device was then inserted into the air gap of the electromagnet. The whole setup was subsequently placed on the stage of a fluorescence microscope (Nikon, ECLIPSE Ti2), where a digital camera (Nikon, DS-Qi2) was mounted. Two precision syringe pumps (Cetoni GmbH, neMESYS 290N) were used to control the flow rates of water $Q_{\mathrm{w}}$ and ferrofluid $Q_{\mathrm{ff}}$. The camera attached to the microscope captured both bright-field images of the particles (showing the distribution of magnetic nanoparticles) and the fluorescent image of the fluorescent dye (showing the distribution of nonmagnetic particles). The experiments were carried out for different flow rate ratios between ferrofluid and water.

\section{NUMERICAL MODELLING}

A three-dimensional (3D) numerical model was implemented to provide more insight into the physics of magnetofluidic spreading in the circular chambers. The parabolic velocity distribution along the channel height leads to a concentration distribution well-known as the "butterfly effect" (Ayodele et al. 2009). Due to the lower velocity and the dominant diffusion over convection, the magnetic force spreads the ferrofluid more conveniently near the walls compared to the centre of the channel. Given the notable distribution of magnetic nanoparticles along the depth direction, a 3D numerical model is required to achieve more accurate results, as the effects of both flow field and magnetic field are considered in three dimensions. 
Figure 1c depicts the model of the microchannel used for the numerical simulation. The ferrofluid enters from the middle inlet with a flow rate of $Q_{\mathrm{ff}}$. DI water enters through the side inlets with a total flow rate of $Q_{\mathrm{w}}$. Under a uniform magnetic field, the magnetic force is most significant at the interface of the two fluids due to the concentration gradient of the magnetic particles and the susceptibility gradient across the two fluids.

The fluid dynamics is modelled by solving the incompressible Navier-Stokes equation (Bird et al. 2001):

$$
\rho \frac{\partial \mathbf{u}}{\partial t}+\rho(\mathbf{u} . \nabla) \mathbf{u}=-\nabla p+v \nabla^{2} \mathbf{u}+\mathbf{F}_{m}
$$

where $\rho$ is the density of the fluid, $p$ is the pressure, $v$ is the dynamic viscosity and $\mathbf{F}_{m}$ is the magnetic volume force.

Moreover, convective/diffusive equation is used to model the distribution of the magnetic nanoparticles in the computational domain (Bird et al. 2001):

$$
\frac{\partial c}{\partial t}+\mathbf{u}_{p} \cdot \nabla c=\nabla \cdot(D \nabla c)
$$

where $c$ denotes the dimensionless concentration (the ratio of the local concentration to the maximum concentration at the channel entrance), $D$ is the diffusivity coefficient, and $\mathbf{u}_{p}$ is the local particle velocity. The diffusion coefficient of fluorescent dye is $1 \times 10^{-9} \mathrm{~m}^{2} \mathrm{~s}^{-1}$ and the diffusion coefficient of magnetic nanoparticles is obtained by Eintein's model $D_{f f}=\frac{k_{b} T}{3 \pi v_{f f} d_{f f}}$, where $f f$ means the ferrofluid, $k_{b}$ is the Boltzman constant, $T$ is the absolute tempreture and, and $d$ is the particle diameter (Hejazian et al. 2016; Soltani et al. 2020). The particle velocity $\mathbf{u}_{p}$ consists of two components $\left(\mathbf{u}_{p}=\mathbf{u}+\mathbf{u}_{s}\right)$, the flow field velocity ( $\mathbf{u}$ ) and the slip velocity ( $\mathbf{u}_{s}$ ) that describes the slip motion of the particles due to the induced magnetic force. According to the previous studies (Blums 1995; Blums et al. 1998), the slip velocity of the magnetic nanoparticles is negligible in a weak magnetic field. Hence, the slip motion of particles is neglected in our simulations. 
The magnetic field is described using Maxwell equations for non-conducting media (Liu et al. 2011):

$$
\begin{aligned}
\nabla \times \mathbf{H} & =0 \\
\nabla \cdot \mathbf{B} & =0
\end{aligned}
$$

where $\mathbf{H}$ and $\mathbf{B}$ are the magnetic field strength and the magnetic flux density, respectively. Magnetic flux density can be calculated using the following equation:

$$
\mathbf{B}=\mu_{0}\left(\mathbf{M}_{m}+\mathbf{H}\right)=\mu_{0}\left(\chi_{m}+1\right) \mathbf{H}
$$

where $\chi_{m}$ represents the local susceptibility of the ferrofluid, $\mu_{0}$ is the permeability constant of the vacuum space with the value of $4 \pi \times 10^{-7} N / A^{2}$, and $\mathbf{M}_{m}$ is the local magnetization. Langevin function describes the magnetization as a function of the magnetic field (Erb et al. 2008; Zhu et al. 2011):

$$
M(H)=M_{s} L(\xi H)=M_{s}\left[\operatorname{coth}(\xi H)-\frac{1}{\xi H}\right],
$$

where $M$ is the magnitude of the ferrofluid magnetization, $M_{s}$ is the saturation magnetisation and $\xi=3 \chi_{0} / M_{s}, \mathrm{Ms}_{\mathrm{s}}=11 \mathrm{mT}, \chi_{0}$ is the initial magnetic susceptibility of the ferrofluid, $\chi_{0}=1.51$. The magnetisation vector $\mathbf{M}$ is in the same direction as the applied magnetic field. Taking into account both the Langevin's function (Eq. 6) and the Maxwell equations, the magnetic susceptibility is defined as (Zhu et al. 2011):

$$
\chi_{f f}=\frac{M_{s}}{H}\left[\operatorname{coth}(\xi H)-\frac{1}{\xi H}\right]
$$

Previous studies suggested that the magnetic susceptibility of the dispersive media linearly depends on the particle concentration for relatively small volume fractions $(\sim 0.2)$ (Sandulyak et al. 2019; Sandulyak et al. 2017). According to these studies, at this concentration the distance between the nanoparticles are sufficiently long to prohibit mutual magnetic effect. The linear dependency of the magnetic susceptibility of the ferrofluid to the volume fraction is fairly valid in this range and volume fraction of 0.02 . Therefore, the local susceptibility is assumed in the present 
work to be a linear function of magnetic particles concentration $\chi_{m}=c \chi_{f f}$. The magnetic scalar potential is introduced as $\mathbf{H}=-\nabla \psi$ that yields (Zhu and Nguyen 2012b):

$$
\nabla \cdot\left(\left(1+\chi_{m}\right) \nabla \psi\right)=0 .
$$

The local magnetic volume force on the fluid is expressed as (Erb et al. 2008; Pankhurst et al. 2003; Rosensweig 1979; Wang et al. 2015a):

$$
\mathbf{F}_{m}=\frac{\chi_{m}}{\mu_{0}}(\mathbf{B} \cdot \nabla \mathbf{B})
$$

The magnetic susceptibility of ferrofluid is large (1.51) compared to that of the water $(-9.0$ $\times 10^{-6}$ ). Therefore, the concentration gradient leads to a significant magnetic flux density gradient and consequently a significant magnetic force (Wang et al. 2015a). As discussed in our previous studies (Zhu and Nguyen 2012a; Zhu and Nguyen 2012b), the existence of a false diffusion in the numerical simulation reduces the sharp concentration gradient across the fluids' interfaces. As such, we employed the second-order discretisation and a smooth and fine mesh to minimise that issue (Bailey 2017).

The magnetic scalar potential is applied to the outer boundaries to determine the magnetic potential and subsequently, the magnetic field inside the domain, Eq. (6), (Zhu and Nguyen 2012b):

$$
\mathbf{H} \cdot \mathbf{n}=-\frac{\partial \psi}{\partial \mathbf{n}}, \forall \mathbf{x} \in \partial \Omega
$$

The variations of density $\rho$ and viscosity $v$ within the domain are determined by using the concept of volume fraction:

$$
\begin{gathered}
\rho=c \rho_{\mathrm{ff}}+(1-c) \rho_{\mathrm{w}} \\
\nu=v_{\mathrm{ff}} e^{(1-c) \ln \left(v_{\mathrm{w}} / v_{\mathrm{ff}}\right)},
\end{gathered}
$$

where the subscripts "w "and "ff" stand for deionised water and ferrofluid, respectively. The densities of ferrofluid and water are 1,100 and $997 \mathrm{~kg} \mathrm{~m}^{-3}$, respectively, and their viscosities are 3 and $1 \mathrm{mPa}$ s, respectively (Wang et al. 2015a). It should be noted that since anionic surfactants 
have been used in the ferrofluid, water is no longer deionised after the diffusion of these surfactants from ferrofluid to the cladding phases.

The mixing index is used to quantitatively evaluate the effect of different parameters on mass distribution within the channel:

$$
\mathrm{MI}=1-\frac{\int_{A}\left|c-c_{\infty}\right| d A}{\int_{A}\left|c_{0}-c_{\infty}\right| d A}
$$

where $c_{0}$ and $c_{\infty}$ represent normalized concentrations of fully unmixed and fully mixed conditions, respectively, and $A$ is the cross-section of the channel.

\section{Results and discussion}

\subsection{Mesh convergence study}

For mesh independence study, concentration mass distribution within a microchannel with one chamber was considered. Free tetrahedral grids were employed in all geometries. Also, a highly smoothed mesh was used in our study to avoid false diffusion. The maximum element growth rate of mesh elements was chosen to be 1.1. Mesh convergence test was performed for four different case studies with a maximum grid size of $6,8,12$, and $16 \mu \mathrm{m}(948,065,476,664,155,835$ and 48,572 number of grids, respectively). The concentration distribution profiles of magnetic particles across the chambers and on the middle depth plane are compared in Figure 2. If the maximum 
mesh size is less than $8 \mu \mathrm{m}$, the changes in concentration distribution is negligible. Therefore, we used a maximum grid size of $6 \mu \mathrm{m}$ in the present study.

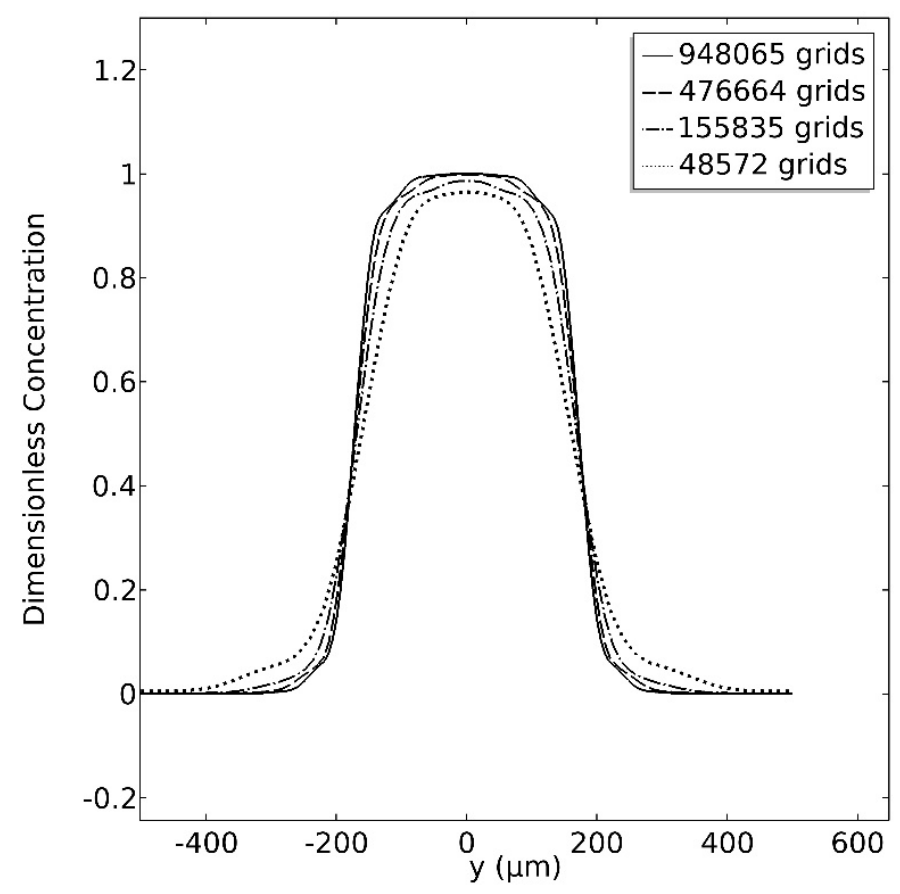

Fig. 2 Comparison of concentration distribution profile of magnetic particles across the chambers and on the middle depth plane, for different numbers of grids.

\subsection{Validation of the numerical simulation with the experimental results}

In this section, the numerical simulation is compared with the experimental data of particle distributions in the first two chambers, with no magnetic field, as well as with a magnetic field of $32.1 \mathrm{mT}$. Figure 3 depicts the top view images of the spreading of magnetic particles and the fluorescent dye. The simulated distribution of magnetic particles qualitatively agrees well with the experimental results. In the fluorescent images, the intensity is maximum (white colour) in the presence of pure fluorescence dye. Also, we observed in the experiments that the core flow deviates to the right side. The main reason for this deviation is related to the variation in cladding flow. Figure 3 shows that the cladding flow is not equally distributed on two sides of the core flow due to the unavoidable fabrication errors, and the left side cladding flow seems to have a larger flow rate compared to the right side. Due to the opaqueness of magnetic nanoparticles, the greyscale intensity is reduced wherever the magnetic nanoparticles overlap the fluorescent dye. In 
contrast, in the simulation, only the variations of the concentration of fluorescent dye are shown, and the influence of overlapping the fluorescent dye with magnetic particles is ignored. The experimental and simulated distributions of the fluorescent dye generally agree; however, there are some partial mismatches particularly for the case of with magnetic field. As observed in Figure $3 \mathrm{~d}(\mathrm{i})$, some regions in the core flow are darker during the experiment. As confirmed in a previous study (Hejazian et al. 2016), the magnetic nanoparticles can have a light-blocking role on the fluorescent intensity. In the darker region, the magnetic nanoparticles on the outer plane have likely blocked the light of the fluorescent dye. Our results confirm that the numerical simulation can predict the spreading behavior of both magnetic nanoparticles and non-magnetic dye.

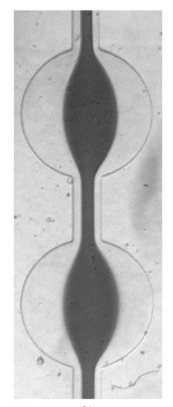

(i)

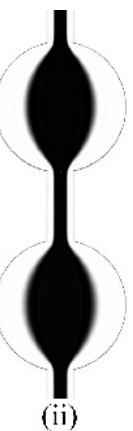

(a)

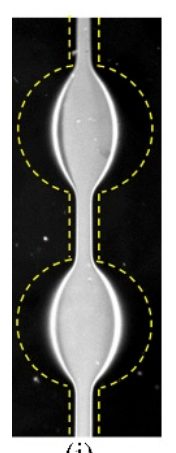

(i)

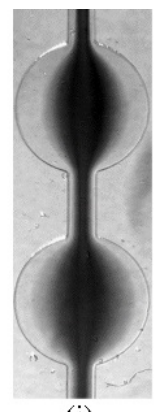

(i)

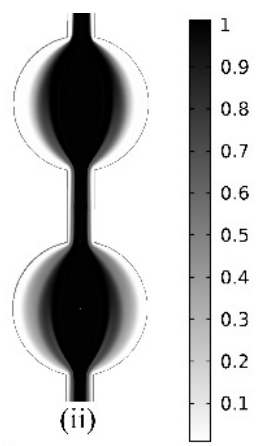

(b)

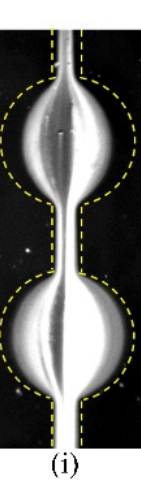

(d)

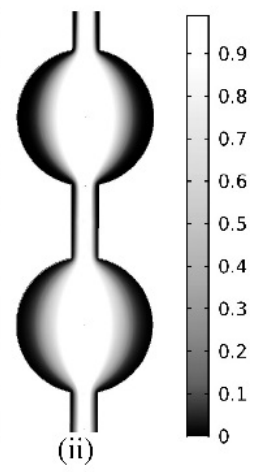

)

Fig. 3 Particle spreading across the consecutive circular chambers: (a) ferrofluid without magnetic field; (b) ferrofluid with a magnetic field; (c) fluorescent dye without magnetic field; (d) fluorescent dye with a magnetic field. All the subsections (i) and (ii) represent the experimental data and numerical results, respectively.

\subsection{Magnetofluidic spreading along different circular chambers without magnetic field}

Figure 4 compares the numerical and experimental spreading phenomenon of ferrofluid in different chambers for the flow ratios $\left(Q_{\mathrm{w}} / Q_{\mathrm{ff}}\right)$ of 1 and $2\left(Q_{f f}=10 \mu \mathrm{m} / \mathrm{min}, Q_{\mathrm{w}}=10\right.$ and $\left.20 \mu \mathrm{m} / \mathrm{min}\right)$. 
As reported in previous studies for a simple rectangular channel without a magnetic field, spreading of the particles relies on the interplay of convection and diffusion (Ayodele et al. 2009). Under the steady-state condition, the interdiffusion region is broadened along the microchannels. A longer axial distance from the inlet corresponds to a more available diffusion time that leads to a wider interdiffusion region. In addition to providing a better visualisation of spreading due to the lateral expansion of fluids, the chambers slow down the flow and enhance spreading. Considering the large ratio of the chamber diameter $d$ to the width $w$ of the straight channel, a series of chambers increases the residence time and enhances spreading.

\section{(a) FF: DI =10ul/min:10ul/min; NO magnet}

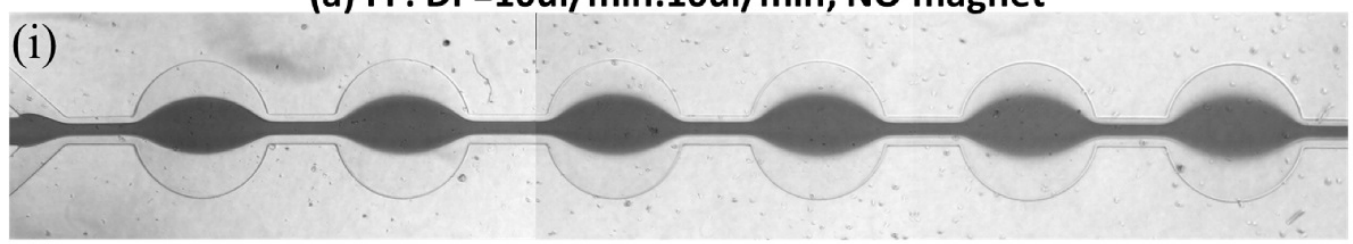

(ii)

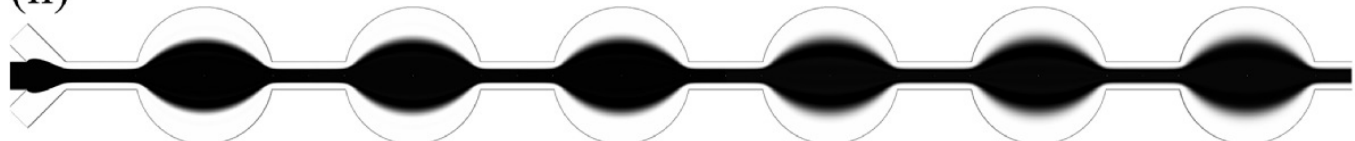

(b) FF: DI =10ul/min:20ul/min; NO magnet

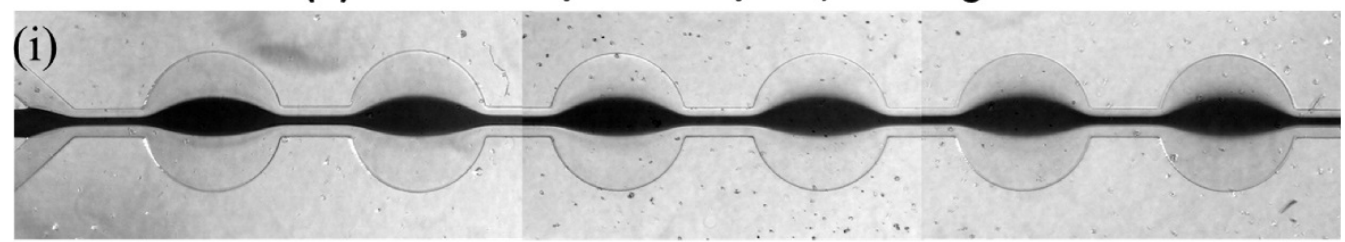

(ii)

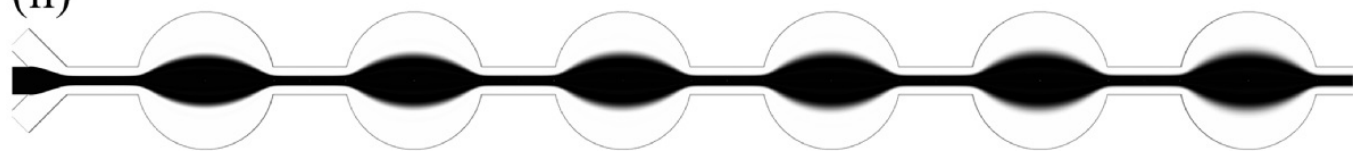

Fig. 4 Spreading of ferrofluid in consecutive circular chambers without magnetic field at flow rate ratios of (a) $1\left(Q_{\mathrm{ff}}=10 \mu \mathrm{l} / \mathrm{min}: Q_{\mathrm{w}}=10 \mu \mathrm{l} / \mathrm{min}\right)$; and (b) 2 (Qff $\left.=10 \mu \mathrm{l} / \mathrm{min}: \mathrm{Qw}=20 \mu \mathrm{l} / \mathrm{min}\right)$. Part (i) is the experimental bright-field results, and part (ii) is the corresponding numerical results.

Due to the relatively small diffusion coefficient of magnetic particles, the transport of magnetic particles occurs at very large Péclet numbers $\left(\mathrm{Pe}=W U / D=1.51 \times 10^{5}\right.$ and $2.65 \times 10^{5}$, where $W$ is the width of the channel, $U$ is the average velocity in the channel, and $D$ is the diffusion coefficient of magnetic particles). In the absence of a magnetic field, spreading is caused by molecular diffusion. In a system with a large Peclet number, spreading is insignificant (Ayodele et al. 2009). As such, we fixed the core flow and varied the cladding flow to examine the different flow rate ratios. Increasing the cladding flow rate narrows the core stream and increases the 
advection of the particles. Thus, lateral (y-direction) spreading of the particles becomes less significant. Figure 5 depicts the depth-averaged concentration profiles of magnetic particles across the different chambers. Because the diagrams are related to the average concentration distribution and the spreading is partial, the progressing spreading is not very clear in Fig. 5. Cross-sectional views of the concentration distribution provide a better insight into the dispersion mechanism, Figure 6.

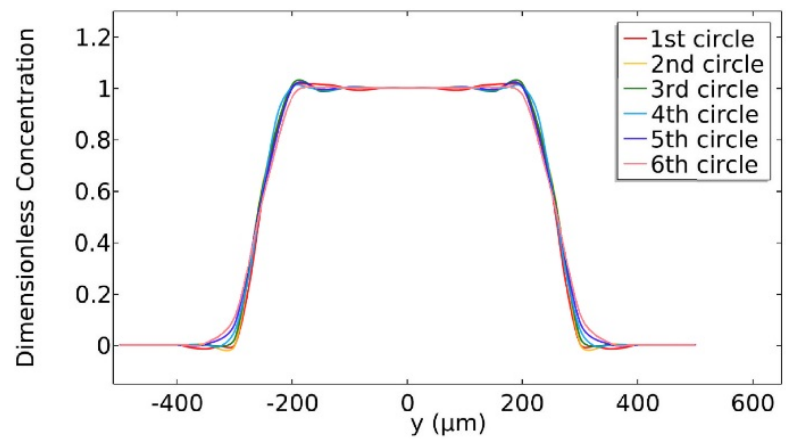

(a)

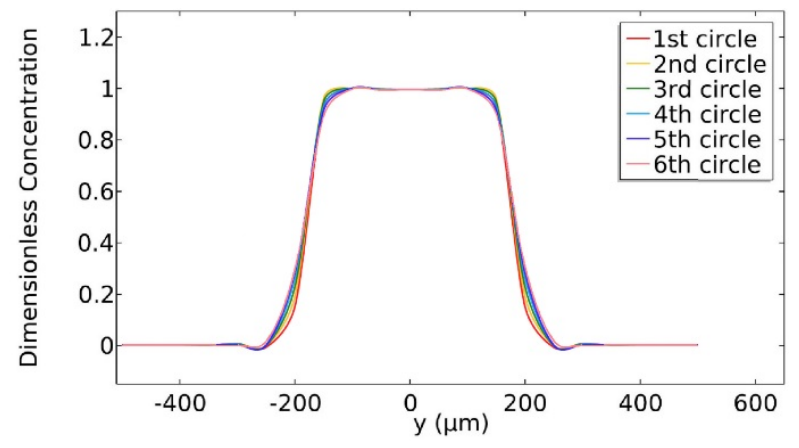

(b)

Fig. 5 Numerical results of depth-averaged concentration distribution profile of magnetic particles across the chambers on the top wall, for the flow rate ratio of (a) 1 ( $\left.Q_{\mathrm{ff}}=10 \mu \mathrm{l} / \mathrm{min}: Q_{\mathrm{w}}=10 \mu \mathrm{l} / \mathrm{min}\right)$; and (b) $2\left(Q_{\mathrm{ff}}=10 \mu \mathrm{l} / \mathrm{min}: Q_{\mathrm{w}}=20 \mu \mathrm{l} / \mathrm{min}\right)$.

As it is discussed in a previous study (Wang et al. 2015b) and observed from Figure 5, due to a hydrodynamic focusing effect, the width of the core flow is narrowed. Narrowing the core flow width while its flow rate is fixed leads to a reduction the residence time of nanoparticles in the channel. Therefore, increasing the flow rate ratio decreases the transversal diffusion of particles in the core flow. The concentration profile of the magnetic particles indicates the "butterfly-like" shape, as described by Ayodele et al. (Ayodele et al. 2009). Considering the no-slip boundary condition at the walls, the parabolic velocity profile results in different residence time of particles across the channel height. The particles close to the top and bottom walls are almost stationary, while those particles at the channel centre are transported with the maximum velocity. Stronger diffusive transport at the wall results in the butterfly-shaped concentration profile (Kamholz et al. 1999). As the flow enters the chambers, the sudden reduction of the axial velocity increases the residence time of the particles. Thus, the chambers can facilitate the homogenisation of the depthwise concentration and smooth out the depth-wise profile of the axial velocity. Increasing the flow rate ratio has a negative impact on the homogenisation of depth-wise concentration. Increasing the 
cladding flow sharpens the velocity profile and increases the difference of the residence time of the particles in the depth direction.

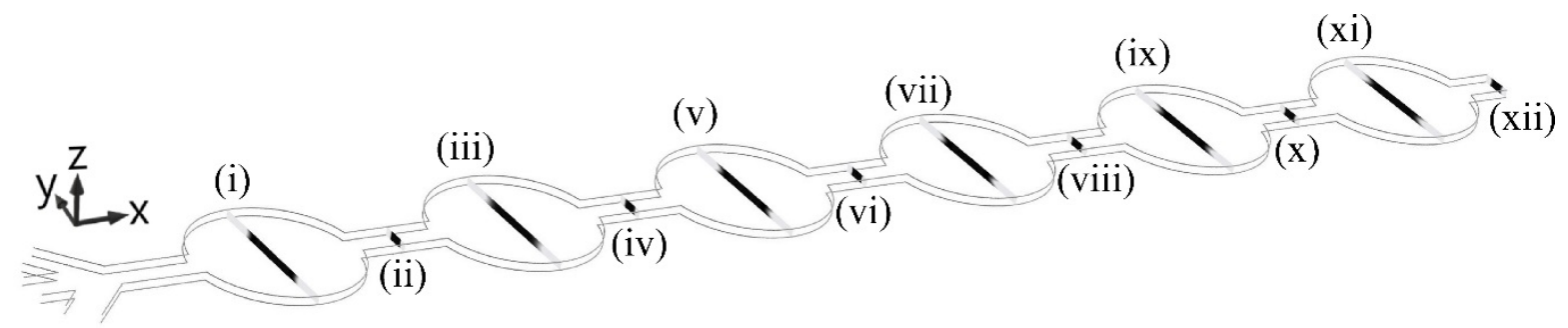

(a)

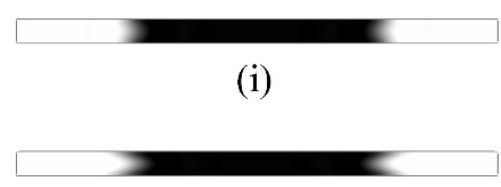

(v)

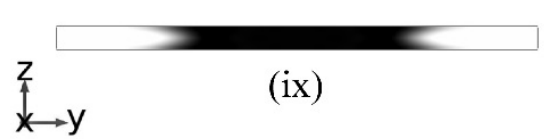

(b)

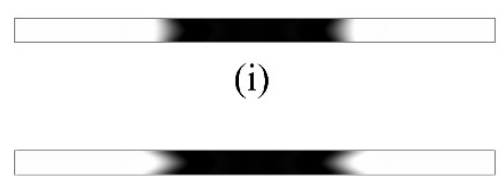

(v)

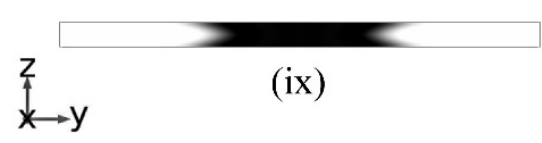

(ii)

(ii)

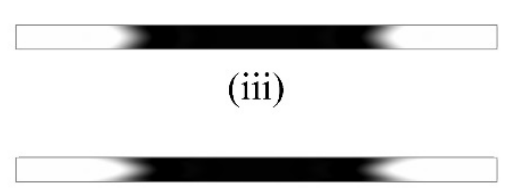

(vi)

$(\mathrm{x})$

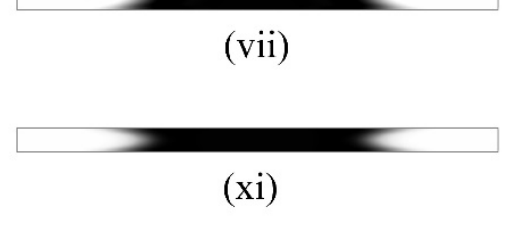

(vi)

(x)

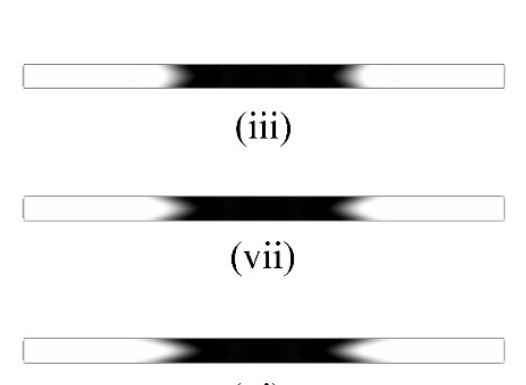

(xi)
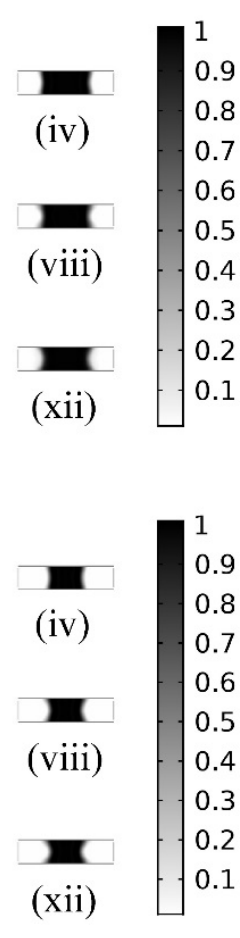

Fig. 6 Numerical results of cross-sectional views of magnetic particles concentration distribution at different distances from channel entrance for flow ratios of (a) 1 , (b) 2 ( $\left.Q_{\mathrm{ff}}=10 \mu \mathrm{m} / \mathrm{min}\right)$. (i) $x=1,500 \mu \mathrm{m}$, (ii) $x=2,260 \mu \mathrm{m}$, (iii) $\mathrm{x}=3,020 \mu \mathrm{m}$, (iv) $x=3,780 \mu \mathrm{m}$, (v) $x=4,540 \mu \mathrm{m}$, (vi) $x=5,300 \mu \mathrm{m}$, (vii) $x=6,060 \mu \mathrm{m}$, (viii) $x=6,820 \mu \mathrm{m}$, (ix) $x=7,580 \mu \mathrm{m}$, (x) $x=8,340 \mu \mathrm{m}$, (xi) $x=9,100$ $\mu \mathrm{m}$, and (xii) $x=9,860 \mu \mathrm{m}$.

\subsection{Magnetofluidic spreading along the circular chambers in a uniform magnetic field}

Figures 7 and 8 show the simulated and experimental results ( $\mathrm{x}-\mathrm{y}$ plane) of magnetofluidic spreading under a uniform magnetic field with different flux densities $\left(Q_{\mathrm{ff}}=10 \mu \mathrm{m} / \mathrm{min}\right.$, $Q_{\mathrm{w}}=10 \mu \mathrm{m} / \mathrm{min}$, the magnetic flux density ranges from 0 to $32.1 \mathrm{mT}$ ). As expected, both results indicate that the uniform magnetic field enhances spreading. Under a uniform magnetic field, a 
magnetic force acts at the fluids interface due to the concentration gradient of the magnetic particles and the consequent magnetisation gradient (Maleki et al. 2019). Magnetic particles migrate from the high concentration to the low concentration, e.g. from the core flow toward the cladding flow. Lateral spreading of magnetic particles is mainly regulated by the interaction between the magnetic and the hydrodynamic forces. Under the same flow condition, the stronger magnetic field induces a stronger transversal dispersion. This phenomenon facilitates convective mass transport and increases the mixing index, Figure 7b. Furthermore, the chambers slow down the flow, causing the magnetic force to dominate over the hydrodynamic force. Thus, lateral spreading in a circular chamber is stronger than that in a narrow channel of the same length. The numerical results of magnetofluidic spreading (Figure 7) reveal more details on this behaviour for the densities of magnetic flux.

First, increasing the density of magnetic flux only improves the spreading of magnetic particles at the top and bottom walls, Figure 7c. At the channel inlet, the concentration of magnetic particles in the core stream is almost homogenous in the depth direction. Therefore, under an applied uniform magnetic field, the fluid interfaces experience the same magnitude of magnetic force. However, the non-uniform distribution of the hydrodynamic force results in different responses along the depth of the channel. Due to the small hydrodynamic force near the walls, the ferrofluid reacts rapidly to the magnetic force in these regions. This results in ferrofluid spreading close to the walls, while the ferrofluid is slightly squeezed in the centre plane to fulfil the mass conservation.

Moreover, due to the smaller convective flow, the magnetic force deforms the flow in chambers more conveniently than in the narrow channels. Therefore, secondary flow occurs in the chambers due to the magnetic force. The strong magnetic force accelerates particles spreading in low-velocity zones of the wall vicinity. In a magnetic field, due to the spreading of ferrofluid at the top and bottom walls, the concentration gradient of magnetic particles increases in the depth direction. The concentration gradient creates a bulk magnetic force that induces secondary flow toward the centre plane. A stronger magnetic field induces a more apparent secondary flow.

Figure $7 \mathrm{~d}$ shows the depth-averaged concentration profiles in the chambers for different densities of the magnetic flux. Increasing the flux density enhances the particles transport toward 
the cladding flow. Enhanced spreading is more evident in the downstream chambers, where particles are exposed to the magnetic field for a longer period of time.

(a)

(1)

(2)

(3)

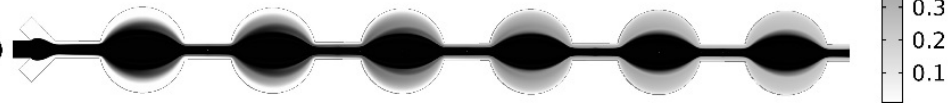

(b)

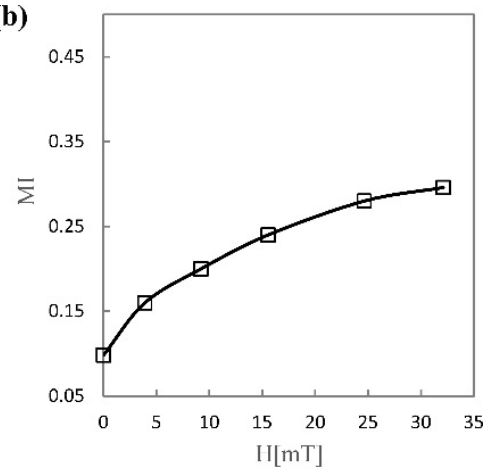

(c)

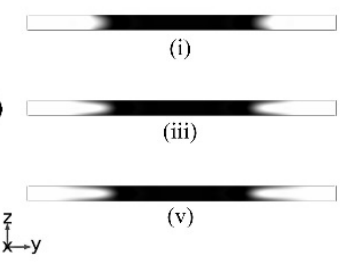

(2)
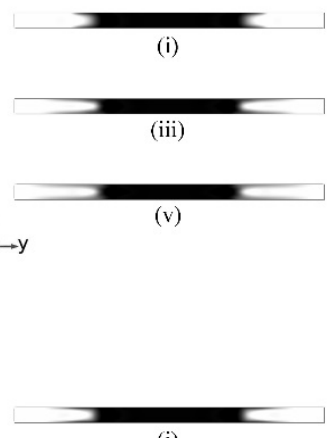

(3)

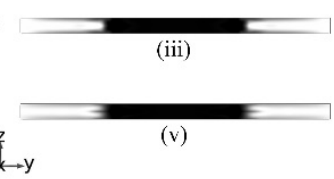

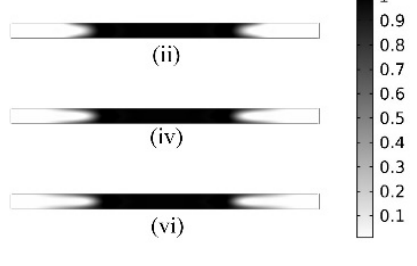

(d)

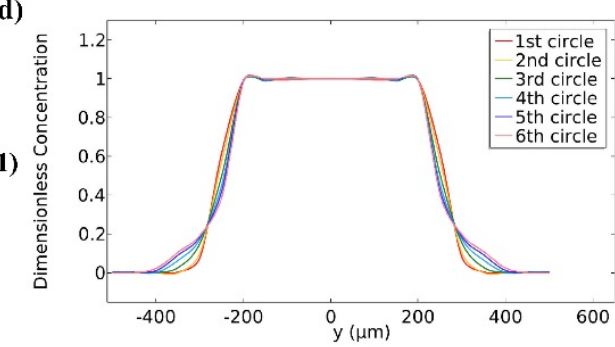

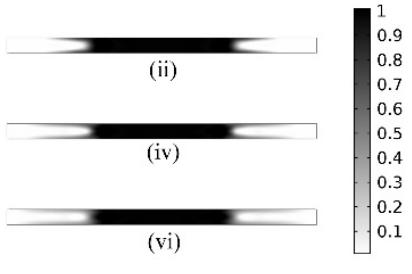
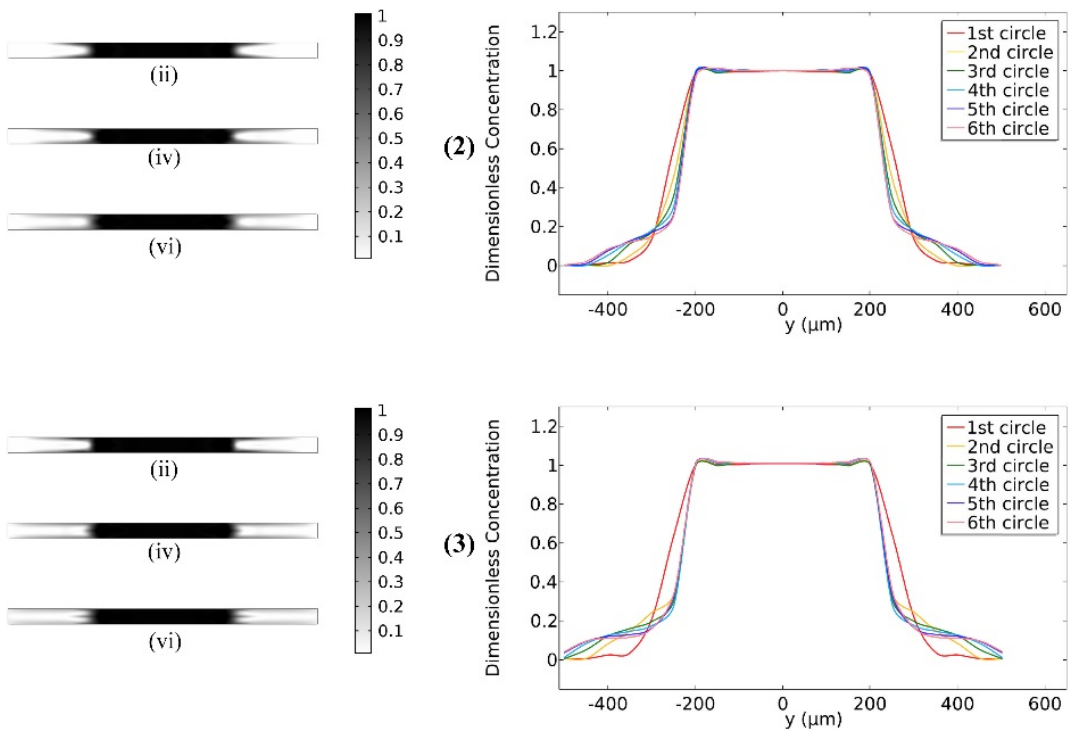

Fig. 7 Spreading of ferrofluid across the consecutive circular chambers (a) top views, (b) mixing index, (c) cross-sectional views, (d) depth-averaged concentration distribution profile of magnetic particles across the chambers, for magnetic fields of (1) $3.91 \mathrm{mT}$, (2) $15.54 \mathrm{mT}$, and (3) $32.10 \mathrm{mT}$ ( $\mathrm{Q}_{\mathrm{ff}}=10 \mu \mathrm{m} / \mathrm{min}, \mathrm{Q}_{\mathrm{w}}=10 \mu \mathrm{m} / \mathrm{min}$ ). (i) $\mathrm{x}=1,500 \mu \mathrm{m}$, (ii) $\mathrm{x}=3,020 \mu \mathrm{m}$, (iii) $\mathrm{x}=4,540 \mu \mathrm{m}$, (iv) $\mathrm{x}=6,060 \mu \mathrm{m}$, (v) $\mathrm{x}=7,580 \mu \mathrm{m}$, (vi) $\mathrm{x}=9,100 \mu \mathrm{m}$. 

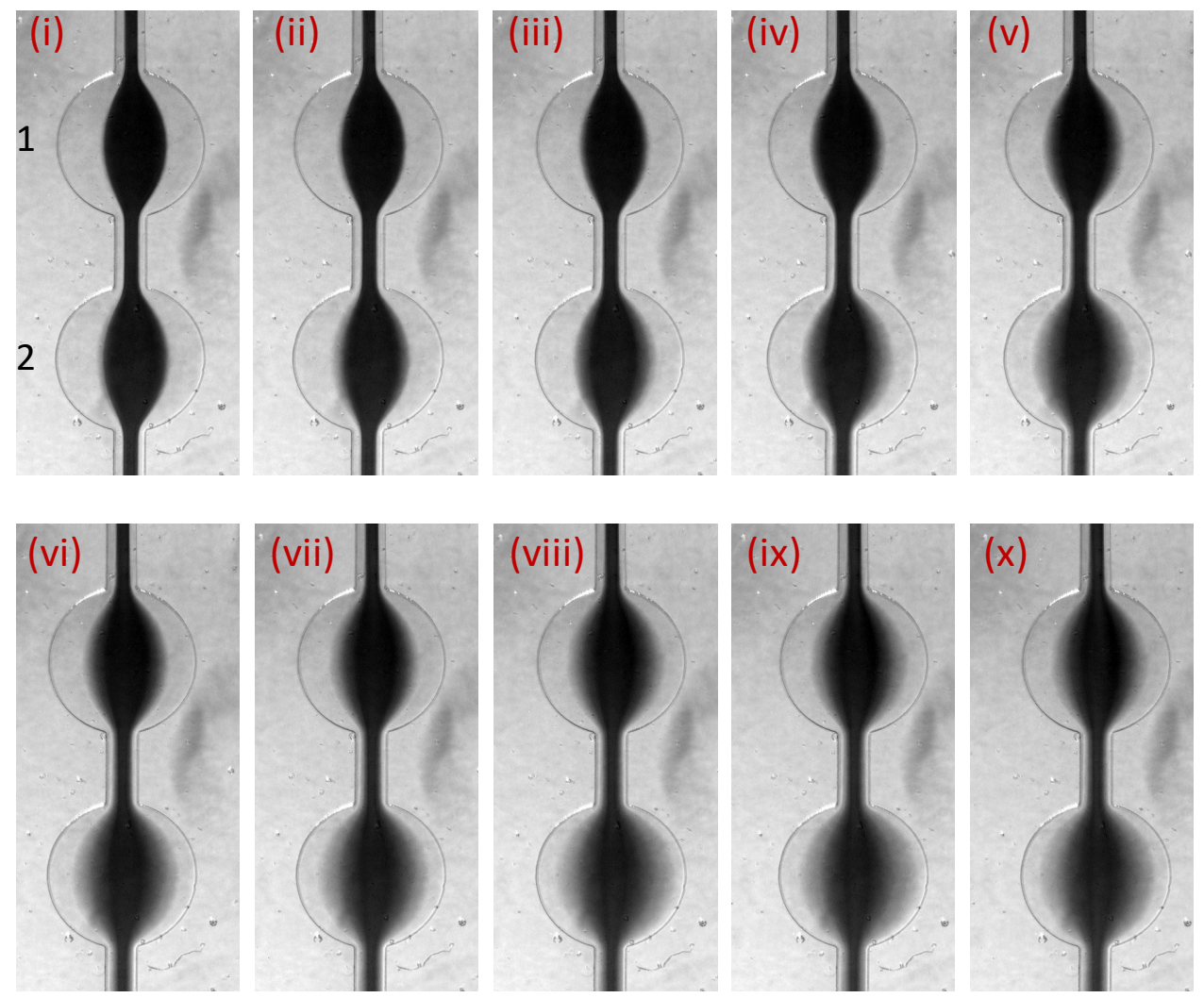

Fig. 8 Experimental results of ferrofluid spreading under magnetic flux densities of (i) $2.17 \mathrm{mT}$, (ii) $3.91 \mathrm{mT}$, (iii) $6.23 \mathrm{mT}$, (iv) $9.2 \mathrm{mT}$, (v) $11.59 \mathrm{mT}$, (vi) $15.54 \mathrm{mT}$, (vii) $20.66 \mathrm{mT}$, (viii) 24.63 $\mathrm{mT}$, (ix) $28.55 \mathrm{mT}$, and (x) $32.1 \mathrm{mT}$. The experimental data are limited to the first two circular chambers. $\left(Q_{\mathrm{ff}}=10 \mu \mathrm{m} / \mathrm{min}, Q_{\mathrm{w}}=10 \mu \mathrm{m} / \mathrm{min}\right)$.

For a better understanding of the spreading mechanism, the superimposed streamlines of the velocity on the contours of magnetic particle concentration are shown in Figure 9a $(H=32.10 \mathrm{mT})$. Additionally, Figure $9 \mathrm{~b}$ shows the contours of the magnetic force over the same cross-sections of the chambers. Given the symmetric flow and forces, only the right half of the cross-sections are illustrated. It is noted that two pairs of counter-rotating flow emerge in the cross-sections, one is near the fluid interface (right pair) and the other is inside the ferrofluid (left pair). After spreading of the magnetic particles in the entrance of the channel, the region of fluid interface turns to a curved shape at the cross-section, with maximum and minimum concentration gradients at the centre and edges, respectively (Ismagilov et al. 2000).

Consequently, the generated magnetic force field at the interface pulls the ferrofluid towards the top and bottom walls, where the hydrodynamic forces are minimum. This behaviour creates 
secondary flows near the walls. As the ferrofluid passes through the chambers with a decreasing concentration gradient near the walls, the magnetic force directs the particles towards the centre. With a more homogenous distribution of magnetic particles, secondary flows become smaller. Furthermore, from the middle of each chamber, the fluid flow shifts from expansion to contraction.

(1)

(2)

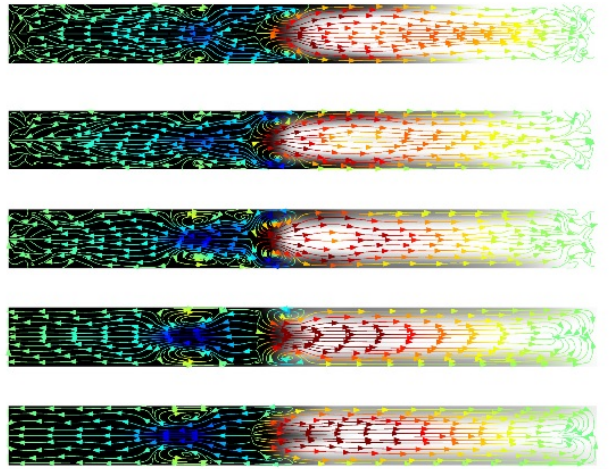

(6)
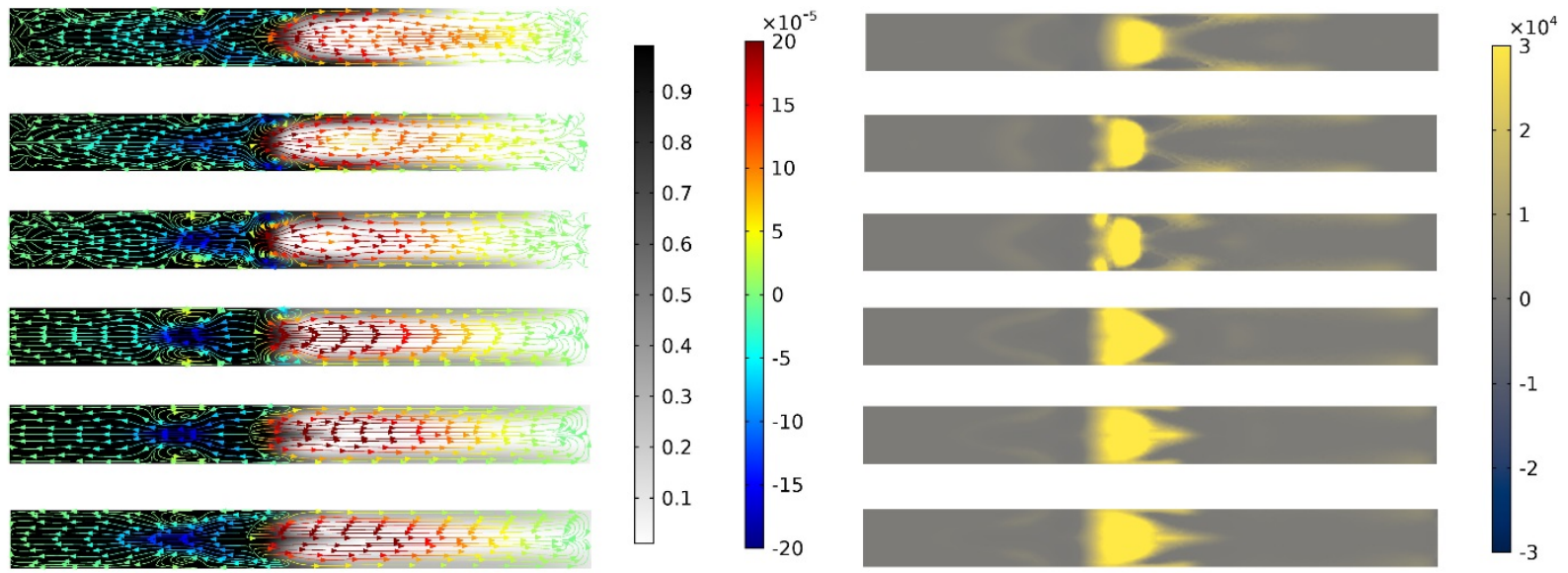

$x_{x \rightarrow y}$

(a)

(b)

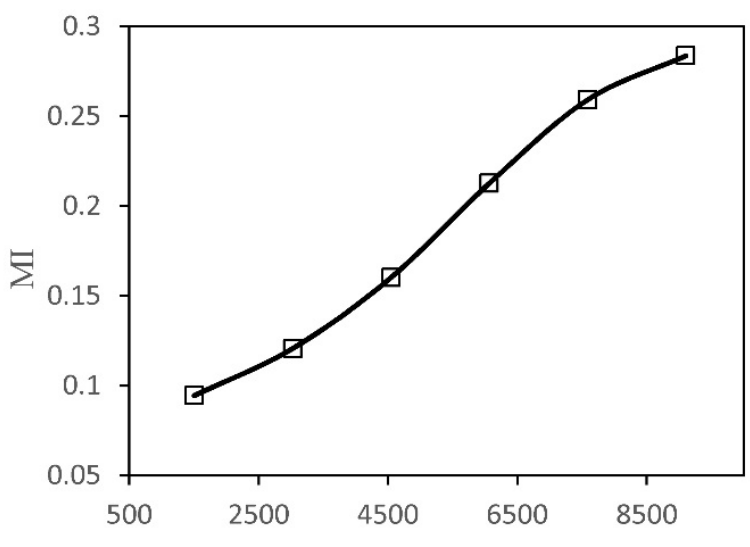

Distance from the microchannel entrance $(\mu \mathrm{m})$

(c)

Fig. 9 Spreading of ferrofluid along the consecutive circular chambers in a uniform magnetic field of $32.10 \mathrm{mT}$. (a) Superimposed streamlines of velocity on the concentration distribution (the grayscale colour bar relates to the magnetic particles concentration and the rainbow colour bar relates to the streamlines of velocity in $\mathrm{y}-\mathrm{z}$ plane); (b) Contours of the magnetic force over the 
same cross-sections of the chambers (c) Mixing index over chambers $\left(Q_{\mathrm{ff}}=10 \mu \mathrm{m} / \mathrm{min}, Q_{\mathrm{w}}=10\right.$ $\mu \mathrm{m} / \mathrm{min})$.

\subsection{Magnetofluidic spreading along microchannels with different chamber geometries in a uniform magnetic field}

Figure 10 represents the numerical results of magnetofluidic spreading within microchannels with different chambers. According to the results, mixing efficiency is slightly improved when the chambers are used in the channel, while there is a negligible difference between the mixing efficiencies for two cases of rectangular and circular chambers. When fluid passes through the chamber, its velocity decreases as the cross-sectional area decreases. Therefore depth-wise variation of flow velocity decreases in chambers, which ameliorates the homogenisation of the particle spreading in depth-direction. Besides, as discussed in the previous section, with a decline in flow velocity, the effects of the magnetic force on the mass transport of the particles become more significant. These behaviours improve overall mass transport in the channel and enhance the mixing efficiency.

Moreover, the geometry of the chambers and the Reynolds number are two effective factors in appearance and the size of the recirculation zones in chambers. Previous studies show that large Reynolds numbers and large ratio between the widths of the chamber and the channel can lead to the growth of recirculation zones within the chamber (Bouquain et al. 2012). Also, in chambers with a sudden change in cross-section, such as rectangular chambers, recirculation zones are generated with lower Reynolds numbers compared to the chambers with a smooth transition in cross-section, such as circular chambers. 
(a)

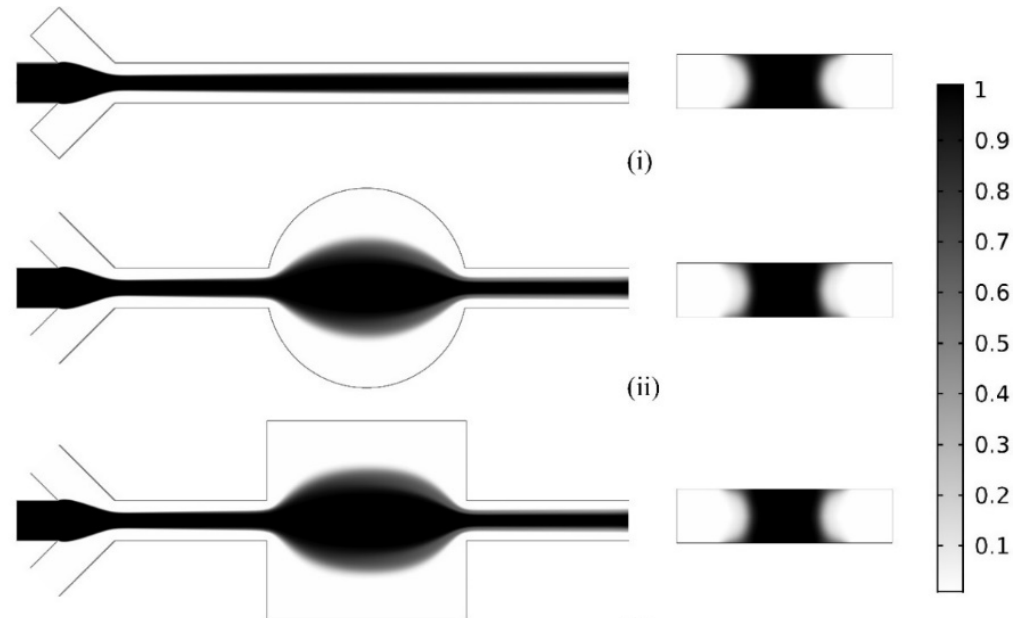

(iii)

(b)

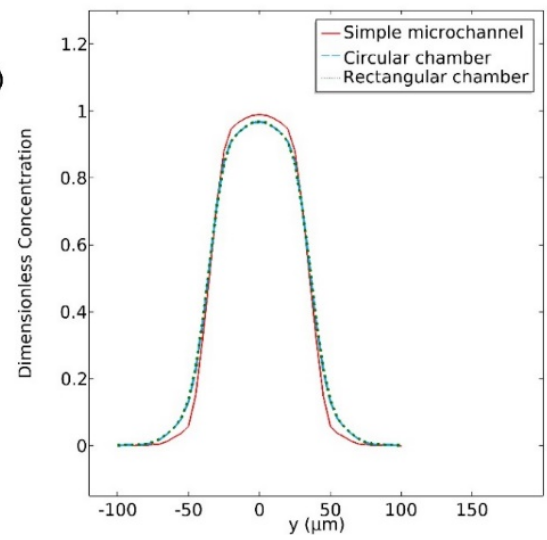

(c)

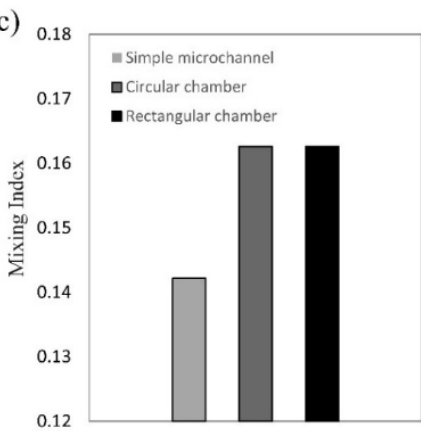

Fig. 10 Spreading of ferrofluid across the microchannels with different chambers geometries: (a) Top view (left side) and a cross-sectional view (right side) of concentration distribution within the (i) simple microchannel, (ii) microchannel with the circular chamber, and (ii) microchannel with the rectangular chamber. (b) Profiles of depth-averaged concentration distribution of magnetic particles across the channel outlet. (c) Mixing index at different types of microchannels $\left(\mathrm{H}=32.10 \mathrm{mT}, \mathrm{Q}_{\mathrm{ff}}=10 \mu \mathrm{m} / \mathrm{min}, \mathrm{Q}_{\mathrm{w}}=10 \mu \mathrm{m} / \mathrm{min}\right)$.

\subsection{Spreading of fluorescent dye in the circular chambers}

Next, we investigated the spreading effect of the fluorescent dye under different densities of the magnetic flux. Figure 11 a,c shows the top and simulated cross-sectional images of the fluorescent dye. Figure 12 shows the corresponding experimental data. The experimental evaluation was only carried out for the first two chambers. The opacity of the magnetic nanoparticles inhibits the total emission of fluorescence so that the overlapping regions appear to be darker than pure fluorescence dye (Hejazian et al. 2016). The diffusion coefficient of fluorescent dye is approximately two orders of magnitude larger than that of the magnetic nanoparticles. As a result, in the absence of a magnetic field, the molecules of the fluorescent dye undergo larger transversal spreading compared 
to the magnetic nanoparticles. It is clear from the experimental results that the edges of the core flow are white, while the inner region is relatively darker, Figure 12.

Additionally, the large diffusion coefficient improves depth-wise mass transport of the fluorescent dye. Therefore, the molecules of the fluorescent dye are homogenised along the depth direction. As such, the butterfly-effect is negligible for these particles. Since the fluorescent molecules are much smaller than magnetic nanoparticles, negative magnetophoresis can be neglected. The molecules of the fluorescent dye are mainly transported through both convection and diffusion. However, the experimental data showed that fluorescent dye behaves similar to the magnetic particles. Increasing the density of the magnetic flux increases the spreading of the fluorescent dye. The reason for this phenomenon is the advective transport of fluorescence dye by the induced secondary flow. In a magnetic field, the molecules of the fluorescent dye initially spread across the main flow (at the top and bottom walls), Figure 11c,d. This behaviour leads to depth-wise concentration gradient at the beginning of the channel. Due to the large diffusion coefficient, the concentration distribution of fluorescence dye is rapidly homogenised as the fluid moves downstream. The large diffusion coefficient of fluorescent dye contributes to their larger mass transport and mixing index compared to the magnetic nanoparticles (Figure 11b), which is reinforced by increasing the magnetic field. Figure 11d shows the profile of depth-averaged concentration of fluorescent dye across different chambers and under various flux densities. As shown in the figure, the transversal spreading increases downstream. In addition, the concentration profiles confirm that the magnetic field enhances the migration of fluorescent dye toward the cladding flow. 
(a)

(1)

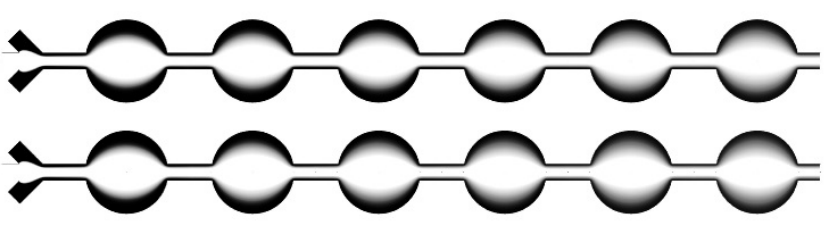

(3)

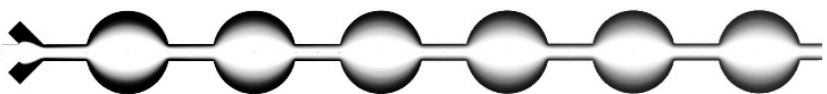

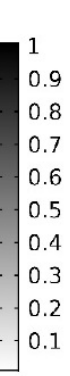

(b)

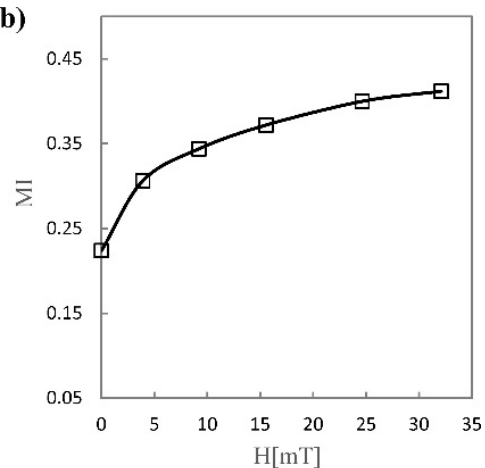

(d) (c)

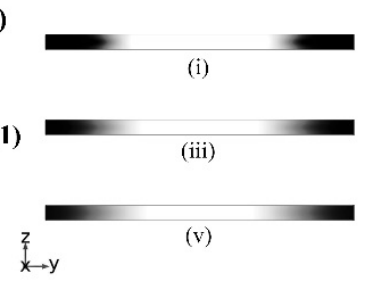

(2)

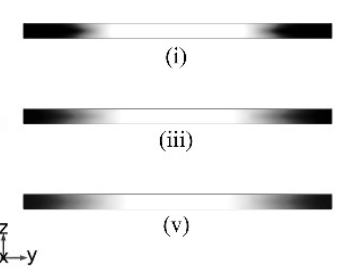

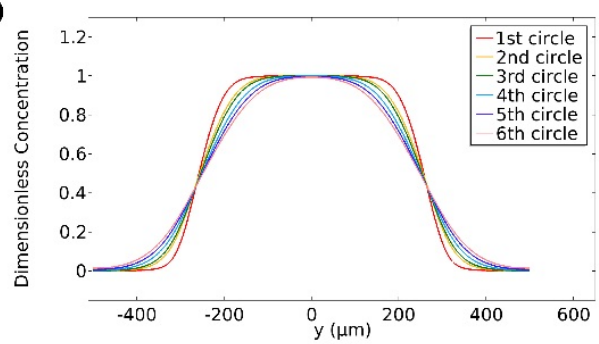
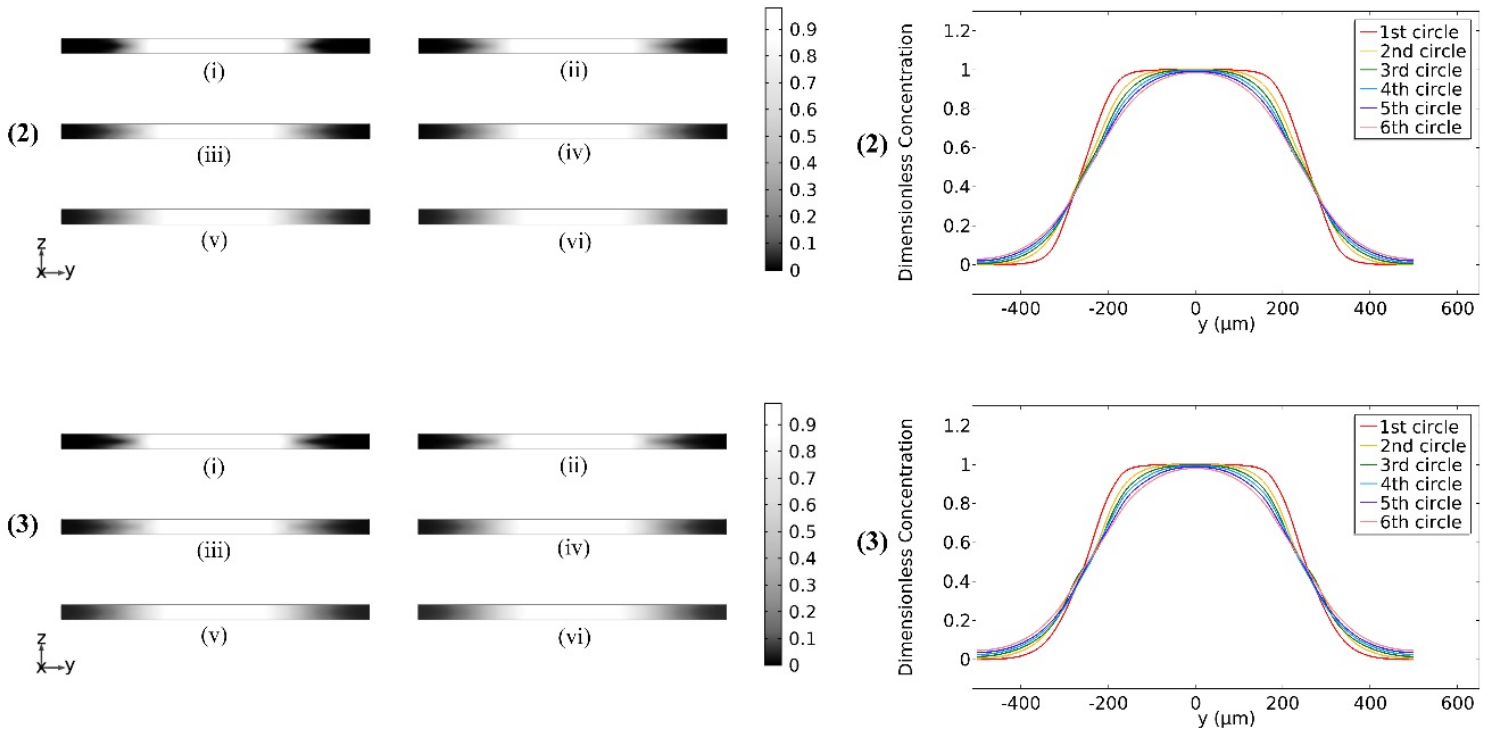

Fig. 11 Spreading of ferrofluid across the consecutive circular chambers (a) top views, (b) mixing index, (c) cross-sectional views, (d) depth-averaged concentration distribution profile of fluorescence dye across the chambers, for magnetic fields of (1) $3.91 \mathrm{mT}$, (2) $15.54 \mathrm{mT}$, and (3) $32.10 \mathrm{mT}\left(\mathrm{Q}_{\mathrm{ff}}=10 \mu \mathrm{m} / \mathrm{min}, \mathrm{Q}_{\mathrm{w}}=10 \mu \mathrm{m} / \mathrm{min}\right.$ ). (i) $\mathrm{x}=1,500 \mu \mathrm{m}$, (ii) $\mathrm{x}=2,260 \mu \mathrm{m}$, (iii) $\mathrm{x}=3,020 \mu \mathrm{m}$, (iv) $x=3,780 \mu \mathrm{m}$, (v) $x=4,540 \mu \mathrm{m}$, (vi) $x=5,300 \mu \mathrm{m}$, (vii) $x=6,060 \mu \mathrm{m}$, (viii) $x=6,820 \mu \mathrm{m}$, (ix) $\mathrm{x}=7,580 \mu \mathrm{m},(\mathrm{x}) \mathrm{x}=8,340 \mu \mathrm{m},(\mathrm{xi}) \mathrm{x}=9,100 \mu \mathrm{m}$, and (xii) $\mathrm{x}=9,860 \mu \mathrm{m}$. 


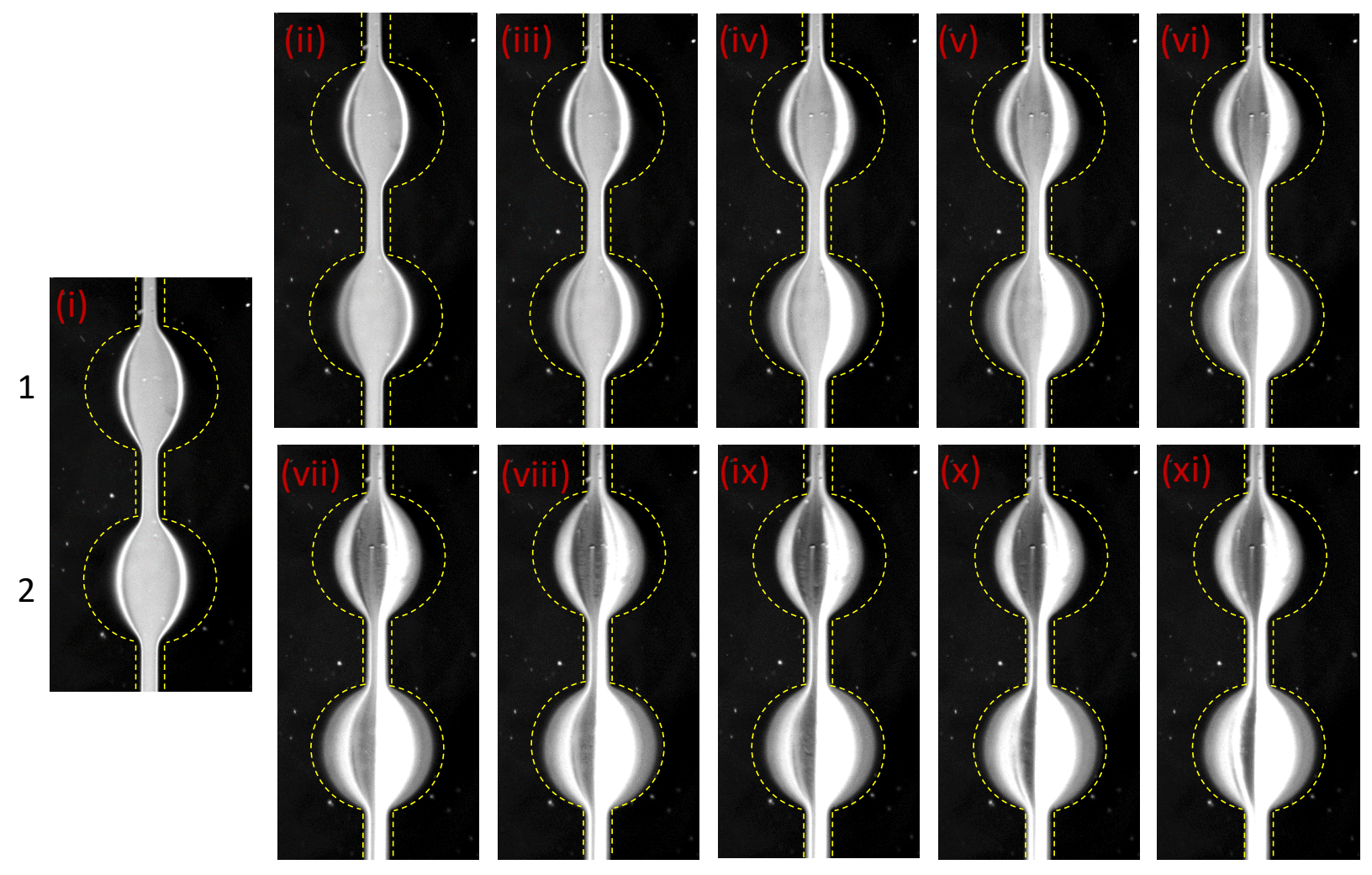

Fig. 12 Experimental results of the spreading of fluorescent dye under different magnetic fields of (i) $0 \mathrm{mT}$, (ii) $2.17 \mathrm{mT}$, (iii) $3.91 \mathrm{mT}$, (iv) $6.23 \mathrm{mT}$, (v) $9.2 \mathrm{mT}$, (vi) $11.59 \mathrm{mT}$, (vii) $15.54 \mathrm{mT}$, (viii) $20.66 \mathrm{mT}$, (ix) $24.63 \mathrm{mT}$, (x) $28.55 \mathrm{mT}$, and (xi) $32.1 \mathrm{mT}$. It should be noted that the fluorescent dye is dissolved within the ferrofluid and it is non-magnetic. ( $Q_{\mathrm{ff}}=10 \mu \mathrm{m} / \mathrm{min}$, $\left.Q_{\mathrm{w}}=10 \mu \mathrm{m} / \mathrm{min}\right)$.

\section{Conclusions}

Magnetofluidic spreading of core flow in circular chambers was investigated experimentally and numerically. The core flow containing ferrofluid and fluorescent dye is sandwiched between two cladding flows of deionized water. Transport phenomena of magnetic nanoparticles and fluorescent dye were investigated for different ratios of flow rate and densities of the magnetic flux. Spreading is controlled by the interplay between the magnetic force, the hydrodynamic force, and molecular diffusion. Under an applied magnetic field, the ferrofluid begins to spread toward the top and bottom walls, where the hydrodynamic force is minimum. The chambers slow down the flow and enhance spreading so that significant mass transfer occurs in the chambers. The numerical simulation revealed that in a magnetic field, cross-sectional secondary flow transports 
particles toward the top and bottom walls. The secondary flow swiftly reduces the concentration gradient of magnetic nanoparticles near the walls. If the magnetic field is strong enough, the pattern of the secondary flow can be observed in downstream chambers. Non-magnetic fluorescent dye was also transported by the transversal secondary flow. Because of the higher diffusion coefficient of the fluorescent particles compared to the larger magnetic nanoparticles, the fluorescent dye is rapidly mixed in depth-wise direction and experiences more significant transversal dispersion. In a magnetic field, the dye molecules generally follow the spreading of magnetic nanoparticles. The larger magnetic force produces a stronger transversal secondary flow that increases transversal transport of fluorescent dye. The results reported here are useful for designing magnetofluidic concentration gradient generators and micromixers.

\section{ACKNOWLEDGEMENT}

The devices were fabricated in the Queensland Microtechnology Facility, part of the Queensland node at Griffith University of the Australian National Fabrication Facility, a company established under the National collaborative Research Infrastructure Strategy to provide nano and microfabrication facilities for Australia's researchers. N.T.N. acknowledges support from the Australian Research Council (ARC) Discovery Project (Grant No. DP180100055).

\section{REFERENCES}

Ayodele SG, Varnik F, Raabe D (2009) Effect of aspect ratio on transverse diffusive broadening: A lattice

Boltzmann study Physical Review E 80:016304 doi:10.1103/PhysRevE.80.016304

Bailey RT (2017) Managing false diffusion during second-order upwind simulations of liquid micromixing 83:940-

959 doi:10.1002/fld.4335

Bird RB, Stewart WE, Lightfoot EN (2001) Transport Phenomena. Wiley,

Blums E (1995) Some new problems of complex thermomagnetic and diffusion-driven convection in magnetic colloids Journal of Magnetism and Magnetic Materials 149:111-115 doi:https://doi.org/10.1016/0304-

\section{$\underline{8853(95) 00350-9}$}

Blums E, Odenbach S, Mezulis A, Maiorov M (1998) Soret coefficient of nanoparticles in ferrofluids in the presence of a magnetic field 10:2155-2163 doi:10.1063/1.869737 
Bouquain J, Méheust Y, Bolster D, Davy P (2012) The impact of inertial effects on solute dispersion in a channel with periodically varying aperture Physics of Fluids 24:083602 doi:10.1063/1.4747458

Chen Y et al. (2014) Rare cell isolation and analysis in microfluidics Lab on a Chip 14:626-645

doi:10.1039/C3LC90136J

D'Eramo L et al. (2018) Microfluidic actuators based on temperature-responsive hydrogels Microsystems \&Amp; Nanoengineering 4:17069 doi:10.1038/micronano.2017.69

https://www.nature.com/articles/micronano201769\#supplementary-information

Diekmann R, Wolfson DL, Spahn C, Heilemann M, Schüttpelz M, Huser T (2016) Nanoscopy of bacterial cells immobilized by holographic optical tweezers Nature Communications 7:13711 doi:10.1038/ncomms13711

https://www.nature.com/articles/ncomms13711\#supplementary-information

Erb RM, Sebba DS, Lazarides AA, Yellen BB (2008) Magnetic field induced concentration gradients in magnetic nanoparticle suspensions: Theory and experiment 103:063916 doi:10.1063/1.2901140

Gao Q-H, Zhang W-M, Zou H-X, Li W-B, Yan H, Peng Z-K, Meng G (2019) Label-free manipulation via the magneto-Archimedes effect: fundamentals, methodology and applications Materials Horizons 6:1359-1379 doi:10.1039/C8MH01616J

Gaster RS et al. (2009) Matrix-insensitive protein assays push the limits of biosensors in medicine Nature Medicine 15:1327 doi: $10.1038 / \mathrm{nm} .2032$

https://www.nature.com/articles/nm.2032\#supplementary-information

Hejazian M, Phan D-T, Nguyen N-T (2016) Mass transport improvement in microscale using diluted ferrofluid and a non-uniform magnetic field RSC Advances 6:62439-62444 doi:10.1039/C6RA11703A

Ismagilov RF, Stroock AD, Kenis PJA, Whitesides G, Stone HA (2000) Experimental and theoretical scaling laws for transverse diffusive broadening in two-phase laminar flows in microchannels 76:2376-2378

doi:10.1063/1.126351

Kamholz AE, Weigl BH, Finlayson BA, Yager P (1999) Quantitative Analysis of Molecular Interaction in a Microfluidic Channel: The T-Sensor Analytical Chemistry 71:5340-5347 doi:10.1021/ac990504j

Kashaninejad N, Nguyen N-T, Chan WK (2012) Eccentricity effects of microhole arrays on drag reduction efficiency of microchannels with a hydrophobic wall Physics of Fluids 24:112004 doi:10.1063/1.4767539 
Knowlton S, Joshi A, Syrrist P, Coskun AF, Tasoglu S (2017) 3D-printed smartphone-based point of care tool for fluorescence- and magnetophoresis-based cytometry Lab on a Chip 17:2839-2851 doi:10.1039/C7LC00706J Kose AR, Koser H (2012) Ferrofluid mediated nanocytometry Lab on a Chip 12:190-196 doi:10.1039/C1LC20864K Liu J, Yap YF, Nguyen N-T (2011) Numerical study of the formation process of ferrofluid droplets Phys Fluids 23:072008 doi:10.1063/1.3614569

Maleki MA, Soltani M, Kashaninejad N, Nguyen N-T (2019) Effects of magnetic nanoparticles on mixing in droplet-based microfluidics 31:032001 doi:10.1063/1.5086867

Marzo A, Seah SA, Drinkwater BW, Sahoo DR, Long B, Subramanian S (2015) Holographic acoustic elements for manipulation of levitated objects Nature Communications 6:8661 doi:10.1038/ncomms9661

https://www.nature.com/articles/ncomms9661\#supplementary-information

Moghadas H, Saidi MS, Kashaninejad N, Nguyen N-T (2018) Challenge in particle delivery to cells in a microfluidic device Drug Delivery and Translational Research 8:830-842 doi:10.1007/s13346-017-0467-3 Munaz A, Shiddiky MJA, Nguyen N-T (2018) Magnetophoretic separation of diamagnetic particles through parallel ferrofluid streams Sensors and Actuators B: Chemical 275:459-469 doi:https://doi.org/10.1016/j.snb.2018.07.176 Nguyen N-T (2012) Micro-magnetofluidics: interactions between magnetism and fluid flow on the microscale Microfluidics and Nanofluidics 12:1-16 doi:10.1007/s10404-011-0903-5

Pankhurst QA, Connolly J, Jones SK, Dobson J (2003) Applications of magnetic nanoparticles in biomedicine Journal of Physics D: Applied Physics 36:R167

Rosensweig RE (1979) Fluid Dynamics and Science of Magnetic Liquids. In: Marton L (ed) Advances in Electronics and Electron Physics, vol 48. Academic Press, pp 103-199. doi:https://doi.org/10.1016/S0065$\underline{2539(08) 60306-6}$

Sandulyak AV, Sandulyak DA, Ershova VA, Sandulyak AA, Polismakova MN (2019) A ferroparticle in a magnetizable disperse medium made up of particles of a like nature: Specific aspects of susceptibility determination Journal of Magnetism and Magnetic Materials 488:165365 doi:https://doi.org/10.1016/j.jmmm.2019.165365 Sandulyak DA, Sandulyak AA, Kiselev DO, Sandulyak AV, Polismakova MN, Kononov MA, Ershova VAJMT (2017) Determining the Magnetic Susceptibility of Ferroparticles from the Susceptibility of Their Dispersive Samples 60:928-933 doi:10.1007/s11018-017-1295-z 
Soltani M, H.H. Tehrani M, Moradi Kashkooli F, Rezaeian M (2020) Effects of magnetic nanoparticle diffusion on microwave ablation treatment: A numerical approach Journal of Magnetism and Magnetic Materials 514:167196 doi:https://doi.org/10.1016/j.jmmm.2020.167196

Tajik P, Saidi MS, Kashaninejad N, Nguyen N-T (2019) Simple, Cost-Effective, and Continuous 3D

Dielectrophoretic Microchip for Concentration and Separation of Bioparticles Industrial \& Engineering Chemistry Research doi:10.1021/acs.iecr.9b00771

van Reenen A, de Jong AM, den Toonder JMJ, Prins MWJ (2014) Integrated lab-on-chip biosensing systems based on magnetic particle actuation - a comprehensive review Lab on a Chip 14:1966-1986 doi:10.1039/C3LC51454D Wang Z, Varma VB, Wang ZP, Ramanujan RV (2015a) Tuning magnetofluidic spreading in microchannels Journal of Micromechanics and Microengineering 25:124001 doi:10.1088/0960-1317/25/12/124001

Wang Z, Varma VB, Xia HM, Wang ZP, Ramanujan RV (2015b) Spreading of a ferrofluid core in three-stream micromixer channels 27:052004 doi:10.1063/1.4919927

Zhang J, Yan S, Yuan D, Zhao Q, Tan SH, Nguyen N-T, Li W (2016) A novel viscoelastic-based ferrofluid for continuous sheathless microfluidic separation of nonmagnetic microparticles Lab on a Chip 16:3947-3956 doi:10.1039/C6LC01007E

Zhao W, Cheng R, Miller JR, Mao L (2016) Label-Free Microfluidic Manipulation of Particles and Cells in Magnetic Liquids 26:3916-3932 doi:10.1002/adfm.201504178

Zhu G-P, Hejiazan M, Huang X, Nguyen N-T (2014) Magnetophoresis of diamagnetic microparticles in a weak magnetic field Lab on a Chip 14:4609-4615 doi:10.1039/C4LC00885E

Zhu G-P, Nguyen N-T (2012a) Magnetofluidic spreading in microchannels Microfluidics and Nanofluidics 13:655663 doi:https://doi.org/10.1007/s10404-012-1056-x

Zhu G-P, Nguyen N-T (2012b) Rapid magnetofluidic mixing in a uniform magnetic field Lab on a Chip 12:47724780 doi:10.1039/C2LC40818J

Zhu G-P, Nguyen N-T, Ramanujan RV, Huang X-Y (2011) Nonlinear Deformation of a Ferrofluid Droplet in a Uniform Magnetic Field Langmuir 27:14834-14841 doi:10.1021/la203931q

Zhu T et al. (2012) Continuous-flow ferrohydrodynamic sorting of particles and cells in microfluidic devices Microfluidics and Nanofluidics 13:645-654 doi:10.1007/s10404-012-1004-9 